\title{
BMJ Open HPV self-sampling acceptability in rural and indigenous communities in Guatemala: a cross-sectional study
}

\author{
Audrey R. Murchland, ${ }^{1}$ Anna Gottschlich, ${ }^{1}$ Kristin Bevilacqua, ${ }^{1}$ Andres Pineda, ${ }^{2}$ \\ Berner Andrée Sandoval-Ramírez, ${ }^{3}$ Christian S Alvarez, ${ }^{1}$ Gina S Ogilvie, ${ }^{4}$ \\ Thomas E Carey, ${ }^{5}$ Mark Prince, ${ }^{6}$ Michael Dean, ${ }^{7}$ Carlos Mendoza Montano, ${ }^{2}$ \\ Alvaro Rivera-Andrade, ${ }^{2}$ Rafael Meza ${ }^{1,8}$
}

To cite: Murchland AR, Gottschlich A, Bevilacqua K, et al. HPV self-sampling acceptability in rural and indigenous communities in Guatemala: a crosssectional study. BMJ Open 2019;9:e029158. doi:10.1136/ bmjopen-2019-029158

- Prepublication history and additional material for this paper are available online. To view these files, please visit the journal online (http://dx.doi. org/10.1136/bmjopen-2019029158).

Received 14 January 2019 Revised 16 August 2019 Accepted 02 September 2019

Check for updates

(C) Author(s) (or their employer(s)) 2019. Re-use permitted under CC BY-NC. No commercial re-use. See rights and permissions. Published by BMJ.

For numbered affiliations see end of article.

Correspondence to

Dr Rafael Meza;

rmeza@umich.edu

\section{ABSTRACT}

Introduction Cervical cancer disproportionately burdens low-income and middle-income countries (LMICs) such as Guatemala. Self-collection testing for human papillomavirus (HPV) has been suggested as a form of cervical cancer screening to facilitate access in LMICs. This study assessed and compared the acceptability of self-collection HPV testing in two rural, indigenous and ethnically distinct communities in Guatemala: Santiago Atitlán, Sololá and Livingston, Izabal.

Methods All participants, women between the ages of 18 and 60 , completed a questionnaire. Eligible participants were also asked to self-collect a vaginal sample and complete a questionnaire regarding comfort and acceptability. Self-collected samples were tested for highrisk HPV using the real-time PCR Hybribio kit.

Results In the indigenous community of Santiago Atitlán, of 438 age-eligible participants, $94 \%$ completed self-collection. Of those, $81 \%$ found it comfortable and $98 \%$ were willing to use it as a form of screening. In the multiethnic (Afro-Caribbean, indigenous) community of Livingston, of 322 age-eligible participants, $53 \%$ chose to self-collect. Among those who took the test, $83 \%$ found it comfortable and $95 \%$ were willing to use it as a form of screening. In Livingston, literacy (can read and/or write vs cannot read or write) was higher in women who chose to self-collect (prevalence ratio 2.25; $95 \% \mathrm{Cl} 1.38$ to 3.68 ). Ethnicity, history of screening and reproductive history were not associated with willingness to self-collect in Livingston. Women in Santiago reported less prior use of healthcare than women in Livingston. Overall, 19\% (106/549) of samples tested positive for high-risk HPV. Conclusion Among women willing to self-collect in rural and indigenous communities in Guatemala, self-collection for HPV testing is highly acceptable. However, willingness to try self-collection might vary across communities and settings. Women from a community that used less healthcare were more likely to choose self-collection. Further research is necessary to determine what factors influence a woman's choice to self-collect.

\section{INTRODUCTION}

Cervical cancer, primarily caused by human papillomavirus (HPV) infection, has a very good prognosis when detected in

\section{Strengths and limitations of this study}

- To our knowledge, little is known about the acceptability of self-collection human papillomavirus testing across the diverse communities within Guatemala and Latin America, and in particular among indigenous populations.

- Our study provided not only a larger sample size compared with previous studies but was also conducted in two differing communities.

- Due to the sensitive nature of the questions related to sexual history, it is possible that a social desirability bias may have resulted in over-reporting of perceived 'good behaviours', such as screening or use of protection, in addition to under-reporting of perceived 'bad behaviours', such as number of lifetime sexual partners and other sexual behaviour measures.

- Sampling methods differed between the two communities due to the lack of reliable census counts in one community, but our sample in this community is reflective of the overall population structure in terms of ethnic, age and other metrics, suggesting that influential selection bias into the study might be limited.

premalignant or early malignant stages. $^{1}$ However, it disproportionately burdens low-income and middle-income countries (LMICs), such as Guatemala, compared with high-income countries (HICs).$^{2-4}$ HICs currently use Pap smears to detect abnormal cervical lesions that can be removed, greatly reducing the risk of cervical cancer. ${ }^{3}{ }^{5}$ However, there are many barriers to implementing successful Pap smear (cytology-based) screening programmes in LMICs, including difficulties establishing sustainable laboratory infrastructure, training and retaining adequate numbers of trained pathologists or cytologists, overburdened primary care clinics, and time and travel limitations for women in reaching screening 
locations. ${ }^{1} 7$ Due to these factors and others, the percentage of women in Guatemala who are screened for cervical cancer remains low; in 2014, only $49.8 \%$ of women (15-49 years of age) reported ever having a Pap smear. Thus, significant improvements in screening or programme implementation are paramount to improving cervical cancer outcomes in Guatemala. ${ }^{389}$

Since more than $90 \%$ of cervical cancers are caused by the HPV virus, HPV testing has been suggested as a possible alternative, primary form of cervical cancer screening. ${ }^{10-12}$ When used in combination with visual inspection with acetic acid (VIA) or Pap smears in low-income settings, HPV testing has been shown to provide significant improvements in the detection of advanced premalignant lesions and cancer in sensitivity as compared with VIA or Pap smear alone, as only women who test positive for HPV need to follow-up with further screening. ${ }^{13-16}$ Previous studies have also confirmed that HPV self-swab kits are comparable to physician-administered samples in their ability to detect carcinogenic, high-risk HPV. ${ }^{17} 18$ Thus, at-home HPV sample collection, with referral to further screening for those positive for high-risk HPV, may be both more acceptable within low-income communities and more programmatically feasible. ${ }^{619}$ Moreover, a 2015 meta-analysis showed that HPV self-sampling, particularly in opt-in programmes, increased participation in cervical cancer screening programmes. However, further work is needed to evaluate acceptable opt-in programmes for women. ${ }^{20}$

Studies have shown that HPV self-sampling is generally acceptable among women in low and high resource settings, as well as immigrant, rural, vulnerable populations. $^{21-31}$ To our knowledge, however, little is known about the acceptability of self-collection HPV testing across the diverse communities within Guatemala, and in particular among rural and indigenous populations. In a pilot cross-sectional study assessing the acceptability of HPV self-collection among 200 women in the Mayan community of Santiago Atitlán, Guatemala, a self-swab HPV test was found to be a highly acceptable form of screening. ${ }^{32}$ Over $80 \%$ of women said that they preferred using a self-swab kit in their home rather than being screened at a doctors' office. However, this pilot study was limited to a relatively small sample in a single, largely homogeneous community, limiting the generalisability of the results to other rural and indigenous communities in Guatemala. Further research is thus needed to evaluate the acceptability of self-collection testing among more diverse rural and indigenous populations within Guatemala, which is very diverse, with over 23 official languages and many indigenous groups, most but not all descendants from the Mayan civilisation. The purpose of this study was to evaluate risk factors, knowledge and attitudes towards cervical cancer and to further assess and compare the acceptability of self-collection HPV testing in two understudied, rural, ethnically distinct communities in Guatemala: Santiago Atitlán, Sololá and Livingston, Izabal.

\section{METHODS}

\section{Study communities}

Santiago Atitlán, Sololá, is a rural community located on Lake Atitlán, in the southwest region of Guatemala, 75 miles west of Guatemala City. The Tz'utujil, a Mayan descendant ethnic group, inhabits the region, which surrounds Lake Atitlán. The primary language of Santiago's inhabitants is Tz'utujil, and over half of the villagers speak Spanish as a second language. ${ }^{32}$ The majority of women in Santiago Atitlán have at most a primary education. Additionally, as a conservative, religious community, it is highly uncommon for women to either drink or smoke, and women in Santiago Atitlán have previously reported having only one lifetime sexual partner. ${ }^{32}$

Livingston, Izabal, is located on the Caribbean coast of the country and is a rural community, only accessible by boat, that is the primary Garifuna settlement in Guatemala. The Garífuna people are considered a unique ethnic group with their own language, culture and cuisine. Additionally, there are large populations of other ethnic and cultural groups located in Livingston, including Q'eqchi' (Mayan descent), Ladinos (non-Mayan descent) and populations of Indian descent. Most women in Livingston are believed to have at least basic primary education.

\section{Patient and public involvement}

The patients were not involved in the development of the research questions, outcome measures or study design. The patients were also not involved in the recruitment and performance of the study. However, the public, Guatemalan physicians, scientists and community health workers (CHWs) were involved in the development of the question, design, validation, recruitment and conduct of the study. Local CHWs were involved in the validation of the survey and study protocol, recruited participants and conducted the interviews, and assisted in providing test results to patients. Guatemalan physicians contributed to the development of the research question and study design, organised the laboratory testing, led and assisted with CHW training, and provided HPV test results to patients. Local laboratory scientists contributed to the study design and conducted the HPV laboratory testing. The continued collaborations with these team members will be used to disseminate study results to patients and Guatemalan officials via publications, presentations and meetings.

\section{Eligibility and recruitment}

Trained CHWs in both communities actively recruited participants through home visits. All CHWs were bilingual and spoke both Spanish and either Tz'utujil, Q'eqchi or Karif (the language of the Garifuna) depending on the location they were working in. Households in Santiago Atitlán were selected at random using stratified multilevel sampling based on maps and population counts of the communities available through the local municipal office and were kept consistent with previous sampling methods. $^{32}$ Households in Livingston were selected at 
random using convenience sampling due to lack of reliable census data at the neighbourhood level. Sampling methods were, otherwise, kept the same as those in Santiago Atitlán.

Selected households that had at least one woman available between the ages of 18 and 60 were invited to participate in the survey component of the study to assess risk factors for, attitudes towards and knowledge of cervical cancer in these communities. For households with more than one eligible woman willing to participate, the female in the household whose birthday was closest to the date of the interview was enrolled in the study. Exclusion criteria consisted of past hysterectomy or previous cervical cancer. Women between the ages of 25 and 54 were also asked to provide a sample, in accordance with Guatemala's current screening recommendations. ${ }^{33}$ Additionally, pregnant women, women currently menstruating and women who had never been sexually active were also excluded from providing samples but could participate in the survey component. Approximately $62 \%$ and $90 \%$ of eligible women contacted were willing to participate in the study in Santiago and Livingston, respectively. All participants in the study provided both oral and written informed consent prior to participation in the study. The consent was documented by a signature or fingerprint of the participant, the surveyors and a witness to the consent process.

\section{Survey}

Data collection consisted of two main components: the surveys and the HPV self-collection tests. Local CHWs in each community were trained as interviewers in the appropriate techniques and protocols before beginning home visits. Two CHWs visited each randomly selected household together and read a recruitment script to a female household member to determine the household's eligibility. CHWs administered the survey and provided kits to collect HPV samples only to willing, eligible participants. Surveys were administered in private rooms of the participant's house to minimise response bias to sensitive questions.

The survey questionnaire included 153 questions concerning demographics, risk factors for cervical cancer and HPV, self-reported attitudes towards screening, healthcare service use and knowledge of cervical cancer and HPV. The survey was developed from the pilot study survey and validated as part of the CHW and translator training to ensure correct translation and cultural relevancy. ${ }^{32}$ Each survey was administered by the CHWs using electronic tablets and the Qualtrics offline app.

All women who participated in the study were compensated with a voucher for a free Pap smear or VIA at a local health clinic. Women in both communities can access free VIAs (Santiago) or Pap smears (Livingston) in the local public health system, but if they chose to use a private clinic instead of the public clinic, the voucher covered their fees.

\section{HPV self-collected samples}

The HPV samples were collected using HerSwab kits, a self-collection sampling method. ${ }^{32} 3435$ If a participant was willing to provide a sample, instructions and graphical materials were provided and the participant collected the sample in a separate, private room from the CHWs. Participants who collected a sample then completed a short post-sample survey with the CHWs of three questions regarding ease, comfort and acceptability of the sampling method: 'How easy was the self-collection swab?'; 'How comfortable was the self-collection swab?'; and 'Would you be willing to collect a sample every 2-3 years to detect HPV as a form of cervical cancer screening?'.

After collection, samples were kept in small, refrigerated coolers carried by the CHWs until they returned to the main study office at the end of the day where samples were then processed to stabilise sample life. The brush component of the HerSwab kit was cut into a $15 \mathrm{~mL}$ test tube using lab scissors. The lab scissors were sterilised using alcohol and an open flame between each sample. Each tube was filled with $5 \mathrm{~mL}$ of Scope mouthwash using a pipette, and tubes were sealed using a cap and parafilm paper. ${ }^{36}$ Mouthwash is a reliable, low-cost transport medium for DNA samples and was used to reflect likely standard operating procedures of HPV screening programme implementation in Guatemala. ${ }^{37}$ Each sample was labelled with the participant's unique identifier. Time of sample processing and condition of sample were recorded.

Stabilised samples were sent to a molecular biology laboratory at the Institute of Nutrition of Central America and Panama (INCAP) in Guatemala City for testing. Samples were tested using the real-time PCR Hybribio HR-13 kit. ${ }^{38}{ }^{39}$ Samples were processed according to the manufacturers protocol and modified to use a $10 \mu \mathrm{L}$ reaction volume for the real-time PCR and run on an ABI-7000. ${ }^{39}$ After testing, samples were labelled as positive for HR-HPV, negative or, if both the HPV probe and the internal control were negative, inconclusive. If a sample test was inconclusive during the first test, it was run an additional time using a $20 \mu \mathrm{L}$ reaction volume, and if no result was obtained, the test was deemed inconclusive.

\section{Follow-up}

A local CHW provided negative and inconclusive results over the phone or through a home visit. Positive results were provided in-person by a study physician who referred participants to their local community health clinic for follow-up and further cervical cancer screening. All participants who could not be reached at the study conclusion were re-contacted either at 6 months or 1 year to provide them with their results. Although women with negative results were not explicitly recommended to attend the clinic, all participants were encouraged to get screened using the voucher provided at the local clinic to support their engagement with local preventative services. Participants who were found to be positive for advanced lesions as a result of follow-up screening were referred for care 
through the free public health infrastructure in Guatemala, as is currently standard practice. Due to the ongoing nature of the project, data on follow-up screening and care are still in the collection process.

\section{Outcomes and statistical analysis}

Willingness and acceptability of self-collection testing, knowledge of HPV and risk factors were evaluated in both communities and across ethnic groups in Livingston, Izabal.

Willingness was measured as whether or not a woman chose to self-collect a sample to be tested for HPV (actual self-collection). The acceptability of sample collection was only assessed for those who self-collected a sample and was analysed using the post-self-swab survey questions described previously.

A target sample size of 500 per community was determined to be able to detect a $5 \%$ difference in self-sampling acceptability with $80 \%$ power, assuming a 95\% acceptability in Santiago Atitlán based on the pilot.

Due to lower rates of actual self-collection in Livingston, differences between Livingston women willing and those not willing to collect a sample were evaluated using two-sample t-tests for means, $\chi^{2}$ tests for proportions and Fisher's exact test for low cell counts. Most women tried self-collection in Santiago, so we restrict these analyses to Livingston.

The main exposures explored for willingness to try self-collection included literacy, marital status, history of Pap smear or VIA, smoking status, alcohol use (a potential proxy for risky behaviour), intrauterine device (IUD) use, family history of cervical cancer and belief of being at risk for cervical cancer. Statistical analyses were run using log-binomial regression. In model set 1 , the relationship between each exposure and sampling decision was unadjusted for other covariates. In model set 2, models were additionally adjusted for age, ethnicity and number of lifetime sexual partners. In model set 3 , we used stepwise selection to select significant exposure covariates (alpha $=0.05$ ) when adjusting for age, ethnicity and number of lifetime sexual partners. Finally, in model set 4, we included all exposure covariates and the adjustment covariates together in a fully adjusted model. The stepwise selected model was further stratified across ethnic groups to evaluate potential effect modification. Due to high prevalence of literacy in Garifuna and Ladino, these groups were combined for stratification to prevent positivity violations (Q'echchi vs Garifuna or Ladino, reflecting a Mayan descent vs non-Mayan descent comparison).

Analyses were restricted to age-eligible women in Livingston with complete covariate information $(n=134$ or $29.4 \%$ excluded due to age ineligibility and $n=13$ or $3.5 \%$ excluded due to missing covariates). Specifically, we excluded five participants missing ethnicity or classified as other ethnicity, one participant missing marital status, one participant missing regular drinking status, three participants missing number of lifetime sexual partners, one participant missing smoking status, and two participants missing family history of cancer resulting in a final sample size $n=309$ for analyses evaluating willingness to try self-collection.

Covariates were parameterised as able to read and/or write (literate) versus unable to either read or write (illiterate), ever married versus never married, ever had a Pap or VIA versus never, ever smoked versus never smoked, regular drinker versus non-regular drinker, ever had an IUD versus never or do not know/refused, family history of cervical cancer present versus absent, and believe at risk for developing cervical cancer (strongly agree/agree versus neutral, disagree, strongly disagree, or unsure/do not know), continuous age and number of lifetime sexual partners (one versus more than one).

Data cleaning and analyses were carried out using SAS V.9.4.

\section{RESULTS}

In total, 956 women were recruited to participate into the study: 500 women in Santiago Atitlán and 456 women in Livingston. Demographic characteristics differed between the two communities: $69.4 \%$ of the participants in Santiago Atitlán had less than primary education and 96.6\% were of Tz'tujil ethnicity. In contrast, only $33.9 \%$ of the participants in Livingston had less than primary education and three ethnic groups were represented: 41.9\% Q'echchi, 32\% Garifuna and 24.8\% mixed ethnicity (Ladino) (table 1).

Knowledge and attitudes regarding HPV and cervical cancer also differed between the communities. Only $11.8 \%$ of participants in Santiago reported previous knowledge of HPV as compared with $62.7 \%$ of Livingston participants. However, when asked about the seriousness of cervical cancer, most participants in both communities responded 'very' or 'extremely' (74.8\% Santiago and $80.9 \%$ Livingston).

Self-reported history of access to healthcare also appeared higher in Livingston than in Santiago. For example, only $5.0 \%$ of participants in Santiago responded that they had ever been tested for HIV, while $56.4 \%$ of Livingston participants responded that they had been previously tested (HIV data not shown in tables due to low cell counts). Furthermore, statistically significantly higher proportions of women from Livingston reported knowledge of breast examinations, receiving more recent Pap testing and regular use of contraceptives. Additionally, a higher proportion of participants in Livingston consistently reported always using protection during sexual intercourse and using tobacco and alcohol than in Santiago (table 1). Additional comparisons of population characteristics can be found in the online supplementary appendix.

\section{Self-collection willingness}

When participants were asked if they would be willing to self-collect at home, the majority of women in both 
Table 1 General population characteristics among all participants

\begin{tabular}{|c|c|c|c|}
\hline & $\begin{array}{l}\text { Santiago Atitlán } \\
\% \text { (N) or mean (SD) }\end{array}$ & $\begin{array}{l}\text { Livingston } \\
\mathrm{N}(\%) \text { or mean (SD) }\end{array}$ & $P$ value \\
\hline $\mathrm{N}$ & 500 & 456 & \\
\hline Age (years) & $34.78(8.44)$ & 32.97 (10.38) & 0.003 \\
\hline Ethnicity & & & $<0.0001$ \\
\hline Tz'tujil & $96.60 \%(483)$ & 0 & \\
\hline Ladino & $1.80 \%(9)$ & $24.78 \%(113)$ & \\
\hline Q'echchi & 0 & $41.89 \%(191)$ & \\
\hline Other & $1.40 \%(7)$ & $1.32 \%(6)$ & \\
\hline Education & & & $<0.0001$ \\
\hline Less than primary & $69.40 \%(347)$ & $33.92 \%(153)$ & \\
\hline Illiterate (neither read nor write) & $48.60 \%(243)$ & $12.50 \%(57)$ & \\
\hline Literate (either read and/or write) & $51.00 \%(255)$ & $87.28 \%(398)$ & \\
\hline Ever married/united & $97.00 \%(485)$ & $62.50 \%(285)$ & $<0.0001$ \\
\hline Breast examination (heard of) & $14.00 \%(70)$ & $66.45 \%(303)$ & $<0.0001$ \\
\hline Pap (ever) & $66.80 \%(334)$ & $58.11 \%(265)$ & 0.0056 \\
\hline Last Pap & & & $<0.0001$ \\
\hline Never or unknown & $36.40 \%(182)$ & $42.54 \%(194)$ & \\
\hline Less than a year & $19.40 \%(97)$ & $29.39 \%(134)$ & \\
\hline Always or almost always & $10.00 \%(50)$ & $19.96 \%(91)$ & \\
\hline Sometimes & $4.80 \%(24)$ & $11.84 \%(54)$ & \\
\hline Rarely or never & $69.60 \%(348)$ & $47.15 \%(215)$ & \\
\hline Unknown or refused & $15.60 \%(78)$ & $21.05 \%(96)$ & \\
\hline Number of lifetime sexual partners & & & $<0.0001$ \\
\hline One & $90.60 \%(453)$ & $70.83 \%(323)$ & \\
\hline More than one & $6.80 \%(34)$ & $25.88 \%(118)$ & \\
\hline Refused & $2.60 \%(13)$ & $3.29 \%(15)$ & \\
\hline Knowledge of HPV & $11.80 \%(59)$ & $62.72 \%(286)$ & $<0.0001$ \\
\hline Severity of CC & & & $<0.0001$ \\
\hline Not or a little & $2.40 \%(12)$ & $12.28 \%(56)$ & \\
\hline Moderate & $22.80 \%(114)$ & $6.80 \%(31)$ & \\
\hline Very or extremely & $74.80 \%(374)$ & $80.92 \%(369)$ & \\
\hline Willing to vaccinate daughters for HPV if available & & & $<0.0001$ \\
\hline Yes & $69.60 \%(348)$ & $82.24 \%(375)$ & \\
\hline No & $1.00 \%(5)$ & $6.80 \%(31)$ & \\
\hline Do not have daughters & $27.60 \%(138)$ & $8.33 \%(38)$ & \\
\hline Refused & $1.80 \%(9)$ & $2.63 \%(12)$ & \\
\hline
\end{tabular}

CC, cervical cancer; HPV, human papillomavirus; IUD, intrauterine device; VIA, visual inspection with acetic acid . 
Table 2 Acceptability of self-collection HPV tests among age-eligible women (25-54 years of age)

\begin{tabular}{|c|c|c|c|}
\hline & $\begin{array}{l}\text { Santiago Atitlán } \\
\text { \% (N) }\end{array}$ & $\begin{array}{l}\text { Livingston } \\
\% \text { (N) }\end{array}$ & \\
\hline $\mathbf{N}$ & $\begin{array}{l}500 \text { (all participants) } \\
438 \text { (age-eligible) }\end{array}$ & $\begin{array}{l}456 \text { (all participants) } \\
322 \text { (age-eligible) }\end{array}$ & P value ${ }^{\star} \dagger$ \\
\hline HPV knowledge & $10.05 \%(44)$ & $63.98 \%(206)$ & $<0.0001$ \\
\hline Self-reported previous Pap (ever) & $71.46 \%(313)$ & $69.88 \%(225)$ & 0.6348 \\
\hline $\begin{array}{l}\text { Abnormal Pap (ever), among those with } \\
\text { previous Pap }\end{array}$ & $16.61 \%(52)$ & $36.89 \%(83)$ & $<0.0001$ \\
\hline Knowledge of VIA & $6.85 \%(30)$ & $1.86 \%(6)$ & 0.0023 \\
\hline Willing to collect sample at home & $93.38 \%(409)$ & $62.42 \%(201)$ & $<0.0001$ \\
\hline Collected sample & $93.61 \%(410)$ & $52.48 \%(169)$ & $<0.0001$ \\
\hline Prefer home screening & $94.06 \%(412)$ & $44.41 \%(143)$ & $<0.0001$ \\
\hline Prefer self-collection & $91.10 \%$ (399) & $41.61 \%(134)$ & $<0.0001$ \\
\hline $\begin{array}{l}\text { Collected sample, among those who said they } \\
\text { were willing to collect at home }\end{array}$ & $96.82 \%(396)$ & $76.12 \%(153)$ & $<0.0001$ \\
\hline $\mathbf{N}$ & $\begin{array}{l}410 \text { (age-eligible; test-taking } \\
\text { participants) }\end{array}$ & $\begin{array}{l}169 \text { (age eligible; test- } \\
\text { taking participants) }\end{array}$ & $P$ value \\
\hline Comfort of test & & & $\dagger<0.0001$ \\
\hline Comfortable & $81.22 \%(333)$ & $83.43 \%(141)$ & \\
\hline Neutral & $5.85 \%(24)$ & $5.33 \%(9)$ & \\
\hline Uncomfortable & $12.68 \%(52)$ & $7.10 \%(12)$ & \\
\hline Unknown & $0.24 \%(1)$ & $4.14 \%(7)$ & \\
\hline Ease of test & & & $0.0241 \dagger$ \\
\hline Easy & $84.63 \%(347)$ & $83.43 \%(141)$ & \\
\hline Neutral & $4.63 \%(19)$ & $7.10 \%(12)$ & \\
\hline Difficult & $10.49 \%(43)$ & $5.33 \%(9)$ & \\
\hline Unknown & $0.24 \%(1)$ & $4.14 \%(7)$ & \\
\hline Willingness to retake test & $98.05 \%(402)$ & $95.27 \%(161)$ & 0.3711 \\
\hline Unknown & $1.46 \%(2)$ & $4.73 \%(8)$ & \\
\hline
\end{tabular}

${ }^{*} \mathrm{P}$ values for means calculated using two-sample t-test; proportions using $\chi^{2}$ test.

†Fisher's exact test used to account for low cell counts.

HPV, human papillomavirus; VIA, visual inspection with acetic acid.

communities responded they would be willing $(93.4 \%$ in Santiago and $62.4 \%$ in Livingston, table 2). However, a lower percentage of women in Livingston actually tried self-collection sampling (93.6\% in Santiago and $52.5 \%$ in Livingston, table 2 and figure 1), as opposed to simply stating willingness in the survey.

We evaluated factors that affected the willingness to try self-collection testing in Livingston. Literacy, the use of health services and beliefs regarding cervical cancer differed between age-eligible women who self-collected a sample compared with those who did not (table 3). Additionally, $31.4 \%$ of the women who ended up not providing a sample had responded previously in the questionnaire that they indeed would be willing to collect a self-swab sample at home. While data are unavailable regarding how many age-eligible women were ineligible to collect a sample due to menstruation or pregnancy, this likely does not entirely account for all women who ultimately chose not to self-collect. Characteristics of women not willing to collect (both reported in the survey and actual sample collection) can be found in the online supplementary appendix. It is interesting to also note that women from Santiago, who reported less prior use of healthcare, were more likely to self-collect.

Literacy was significantly higher among women who self-collected a sample in Livingston compared with those who did not (crude prevalence ratio (PR) 2.04; 95\% CI 1.27 to 3.28; adjusted PR, 2.25; 95\% CI 1.38 to 3.68) (table 4). IUD use was also higher among women who self-collected a sample in Livingston (crude PR 1.49; $95 \%$ CI 1.15 to 1.94; adjusted PR 1.43; 95\% CI 1.08 to 1.88 ) (table 4). Additionally, regular drinking and never being married were higher among women who self-collected but not significant (regular drinking, crude PR 1.18; 95\% CI 0.95 to 1.48 ; adjusted PR 1.14; $95 \%$ CI 0.89 to 


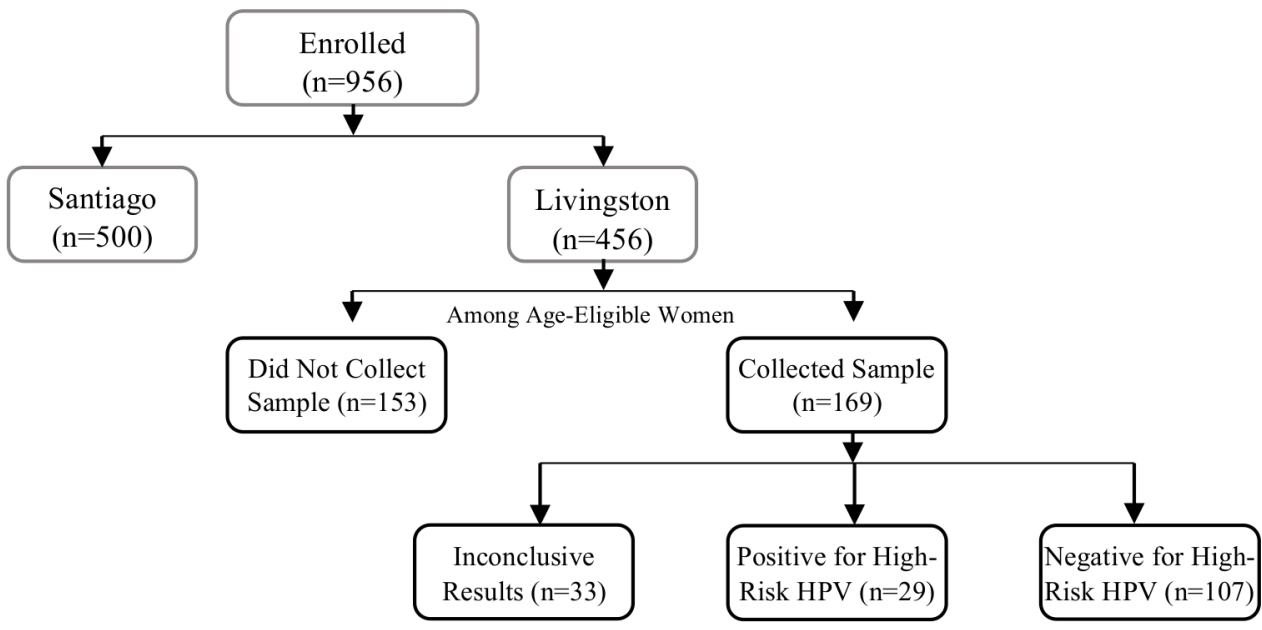

Figure 1 Flow chart of Livingston self-collection sampling and testing. HPV, human papillomavirus.

1.46; never married, crude PR 1.19; $95 \%$ CI 0.96 to 1.48 ; adjusted PR 1.15 ; $95 \%$ CI 0.91 to 1.43 ) (table 4 ).

Using stepwise selection with adjustment for age, ethnicity, and more than one lifetime sexual partner, only literacy was selected as an exposure covariate (PR $2.25 ; 95 \%$ CI 1.38 to 3.68 ). When stratifying ethnic group (Q'echchi vs Garifuna and Ladino), the association between literacy and actual sample collection remained positive (table 5). However, this relationship only remained statistically significant among Q'echchi participants. Interaction terms between ethnic group (Q'echchi vs not) and literacy revealed that the effect of literacy among the Q'echchi was not significantly different from the Ladinos and Garifunas (interaction term PR 0.94; $95 \%$ CI 0.25 to 3.59 ).

Finally, when fully adjusting for all exposure covariates, the effect of literacy continued to remain significant (PR $1.68 ; 95 \%$ CI 1.12 to 2.51 ).

\section{Self-collection acceptability and comfort}

Among those who did collect a sample, the self-collection testing was highly acceptable in both communities. Of Santiago participants who self-collected, $81.2 \%$ found it comfortable and $84.6 \%$ reported that the HerSwab was easy to use. Among Livingston participants who self-collected, $83.4 \%$ found it comfortable and $83.4 \%$ reported it was easy to use. Among those who chose to self-collect, almost all participants in both locations reported that they were willing to use it as a form of cervical cancer screening (98.1\% in Santiago and $95.3 \%$ in Livingston) (table 2).

\section{HPV prevalence}

Overall, $19 \%$ of samples tested positive for high-risk HPV $(n=549)$. Furthermore, $18.7 \%$ of samples from Santiago Atitlán $(n=77)$ tested positive for high-risk HPV and $21.3 \%$ of samples from Livingston $(\mathrm{n}=29)$ tested positive, but this difference was not statistically significant ( $\mathrm{p}=0.4923)$. In total, $94 \%$ of participants who sampled in Santiago Atitlán and $88.5 \%$ of participants who sampled in Livingston were provided with their test results. Overall,
$12.3 \%$ of HPV tests were found to be inconclusive ( $\mathrm{n}=44$ $(9.6 \%))$ from Santiago Atitlán and $n=33$ (19.5\%) from Livingston).

\section{DISCUSSION}

In this study, we assessed the acceptability of HPV self-collection testing as an alternative form of primary cervical cancer screening in indigenous and rural communities in Guatemala. We found that self-collection appears to be highly acceptable among women who tried it, independent of community and ethnicity. Most women reported that self-collection was comfortable and easy to use, and almost all women who tried it reported being willing to use it as a form of cervical cancer screening in the future. These results are consistent with other studies looking at self-collection acceptability both within Guatemala and other LMICs. ${ }^{27}{ }^{32}$ This study was further able to build on previous studies and provide important information regarding HPV self-collection testing acceptability at the community level, and in a community that had not been previously evaluated.

Our study also found, however, that there were differences between communities in willingness to try self-collection. Willingness to try self-collection testing remained consistently high among participants in Santiago Atitlán as reported in the pilot study conducted in 2015 (93\% in 2015 vs $93.6 \%$ in 2016). ${ }^{32}$ In Livingston, however, even among women who first responded in the survey or consent form that they would be willing to collect a sample, actual self-collection was lower. We found that willingness to self-collect in Livingston was consistently associated with higher levels of literacy and prior IUD use. In contrast, ethnicity, history of cervical cancer screening and health behaviours were not associated with willingness to self-collect. Stratified analyses suggested that there were no qualitative differences in the association between literacy and sample collection across ethnic groupings (Mayan descent vs non-Mayan descent) in Livingston. However, high prevalence of literacy among Garifuna 
Open access

Table 3 Population characteristics within Livingston; *age-eligible women who sampled vs age-eligible women who did not sample

\begin{tabular}{|c|c|c|c|}
\hline & $\begin{array}{l}\text { Took the sample } \\
\%(\mathrm{~N}) \text { or mean (SD) }\end{array}$ & $\begin{array}{l}\text { Did not take the sample } \\
\%(\mathrm{~N}) \text { or mean (SD) }\end{array}$ & $P$ value $†$ \\
\hline N & $52.48 \%(169)$ & $47.52 \%(153)$ & \\
\hline Age (years) & $34.98(7.76)$ & $36.35(7.66)$ & 0.1141 \\
\hline Ethnicity & & & 0.6986 \\
\hline Ladino & $50.59 \%(43)$ & $49.41 \%(42)$ & \\
\hline Garifuna & $54.37 \%(56)$ & $45.63 \%(47)$ & \\
\hline Q'echchi & $51.94 \%(67)$ & $48.06 \%(62)$ & \\
\hline Other & $75.0 \%(3)$ & $25.00 \%(1)$ & \\
\hline Declined & 0 & $100.0 \%(1)$ & \\
\hline Education & & & 0.0784 \\
\hline Less than primary & $46.34 \%(57)$ & $53.66 \%(66)$ & \\
\hline Primary or secondary & $57.14 \%(60)$ & $42.86 \%(45)$ & \\
\hline More than secondary & $56.18 \%(50)$ & $43.82 \%(39)$ & \\
\hline Unknown & $40.00 \%(2)$ & $60.00 \%(3)$ & \\
\hline Literacy & & & 0.0005 \\
\hline Illiterate (neither read nor write) & $29.17 \%$ (14) & 70.83\% (34) & \\
\hline Literate (either read and/or write) & $56.57 \%(155)$ & $43.43 \%(119)$ & \\
\hline Married/united & & & 0.2365 \\
\hline Ever & $49.78 \%(112)$ & $50.22 \%(113)$ & \\
\hline Never & $50.22 \%(56)$ & $41.67 \%(40)$ & \\
\hline Unknown & $100.0 \%(1)$ & 0 & \\
\hline Pap or VIA & & & 0.2324 \\
\hline Ever & $54.67 \%(123)$ & $45.33 \%(102)$ & \\
\hline Never & $47.42 \%(46)$ & $52.58 \%(51)$ & \\
\hline Ever smoke & & & 0.6309 \\
\hline Ever & $53.33 \%(16)$ & $46.67 \%$ (14) & \\
\hline Never & $52.23 \%(152)$ & $47.77 \%(139)$ & \\
\hline Unknown & $100.0 \%(1)$ & 0 & \\
\hline Regular drinker & & & 0.7619 \\
\hline Yes & $56.67 \%(17)$ & $43.33 \%(13)$ & \\
\hline No & $51.89 \%(151)$ & $48.11 \%(140)$ & \\
\hline Unknown & $100.0 \%(1)$ & 0 & \\
\hline Used IUD & & & 0.0112 \\
\hline Ever & $73.08 \%(19)$ & $26.92 \%(7)$ & \\
\hline Never & $51.37 \%(150)$ & $48.63 \%(142)$ & \\
\hline Do not know & & $100.0 \%(4)$ & \\
\hline Use protection & & & 0.1260 \\
\hline Always or almost always & $58.62 \%(34)$ & $41.38 \%(24)$ & \\
\hline Sometimes & $58.54 \%(24)$ & $41.46 \%(17)$ & \\
\hline Rarely or never & $53.75 \%(86)$ & $46.25 \%(74)$ & \\
\hline Unknown & $39.68 \%(25)$ & $60.32 \%(38)$ & \\
\hline Number of lifetime partners & & & 0.0670 \\
\hline One & $49.12 \%(111)$ & $50.88 \%(115)$ & \\
\hline More than one & $61.29 \%(57)$ & $38.71 \%(36)$ & \\
\hline
\end{tabular}

Continued 
Table 3 Continued

Took the sample

$\%$ (N) or mean (SD)
Did not take the sample $\%$ (N) or mean (SD)

P value†

$66.66 \%$ (2)

$44.17 \%(91)$

0.1097

$55.83 \%(115)$

$36.36 \%(4)$

$56.52 \%(13)$

$52.58 \%(102)$

$48.61 \%(35)$

$55.14 \%(102)$

$48.91 \%(67)$

0.4191

\begin{tabular}{|c|c|c|c|}
\hline Not & $36.36 \%(4)$ & $63.64 \%(7)$ & \\
\hline A little & $68.18 \%(15)$ & $31.82 \%(7)$ & \\
\hline Moderate & $56.52 \%(13)$ & $43.48 \%(10)$ & \\
\hline Very & $52.58 \%(102)$ & $47.42 \%(92)$ & \\
\hline Extremely & $48.61 \%(35)$ & $51.39 \%(37)$ & \\
\hline Believe at risk of CC & & & 0.2684 \\
\hline Strongly agree or agree & $55.14 \%(102)$ & $44.86 \%(83)$ & \\
\hline Other & $48.91 \%(67)$ & $51.09 \%(70)$ & \\
\hline $\begin{array}{l}\text { Willing to vaccinate daughters for HPV if } \\
\text { available }\end{array}$ & & & 0.4024 \\
\hline Yes & $54.32 \%(151)$ & $45.68 \%(127)$ & \\
\hline No & $47.62 \%(10)$ & $52.38 \%(11)$ & \\
\hline Do not have daughters & $38.46 \%(5)$ & $61.54 \%(8)$ & \\
\hline Refused & $30.00 \%(3)$ & $70.00 \%(7)$ & \\
\hline
\end{tabular}

*\% calculated for sampling decision by each covariate.

†P values for means calculated using two-sample t-test; proportions using $\chi^{2}$ test .

CC, cervical cancer; HPV, human papillomavirus ; IUD, intrauterine device; VIA, visual inspection with acetic acid.

made it difficult to evaluate differences between Ladinos and Garifunas in the association between literacy and sampling decision.

The results suggest that HPV self-collection testing programme implementation may need to target populations based on relative levels of literacy within communities. A previous study examining HPV acceptability and intention in the UK similarly found that low education and self-efficacy, defined as an individual's belief in their capability to exercise control over challenging demands, were associated with low sampling intentions. ${ }^{30}$ In Guatemala, the inability to either read or write in Spanish may negatively influence a woman's perceived self-efficacy and her confidence in navigating public health infrastructure or self-collecting a vaginal sample, particularly if her surrounding community has high levels of literacy. This population would greatly benefit from HPV self-collection testing as a primary form of cervical cancer screening due to its strength in concentrating less accessible and more invasive screening modalities only towards those that are at high risk (ie, positive for HPV). Our results in Livingston suggest that it might be critical that, if implemented, HPV screening and education programmes are tailored such that they are more accessible to low-literacy populations and, thus, increase perceived self-efficacy in navigating the existing public health infrastructure.

High prevalence of self-collection testing in Santiago Atitlán, a community with low literacy levels, as compared with the low rates of self-collection testing among those with low literacy in Livingston may reflect larger community differences in awareness or access to screening modalities rather than a lack of effect of literacy in Santiago or an effect of ethnicity. Although women from Santiago reported slightly higher rates of ever receiving cervical cancer screening than women in Livingston, women in Livingston report much higher rates of recent cervical cancer screening than women in Santiago. Santiago Atitlán remains largely deficit in accessible and affordable cervical cancer screening, while Livingston has regular, public or private, screening campaigns in the community. This difference in general community access and infrastructure, then, may be acting as an effect modifier on the association between literacy and screening between these two communities, suggesting that self-collection might be better received at first in communities that do not have other alternatives, whereas some initial scepticism might be found in places with existing cervical screening programmes, independently of their quality and efficacy. More research is necessary to evaluate if self-efficacy, relative literacy level or general community access to healthcare resources and screening affect women's willingness to try self-sampling HPV testing. However, the high rates of acceptability and willingness to retake among women who self-collected in both communities suggest that once experienced, self-collection is a valid, and even preferred, alternative to other screening modalities from the women's perspective. 


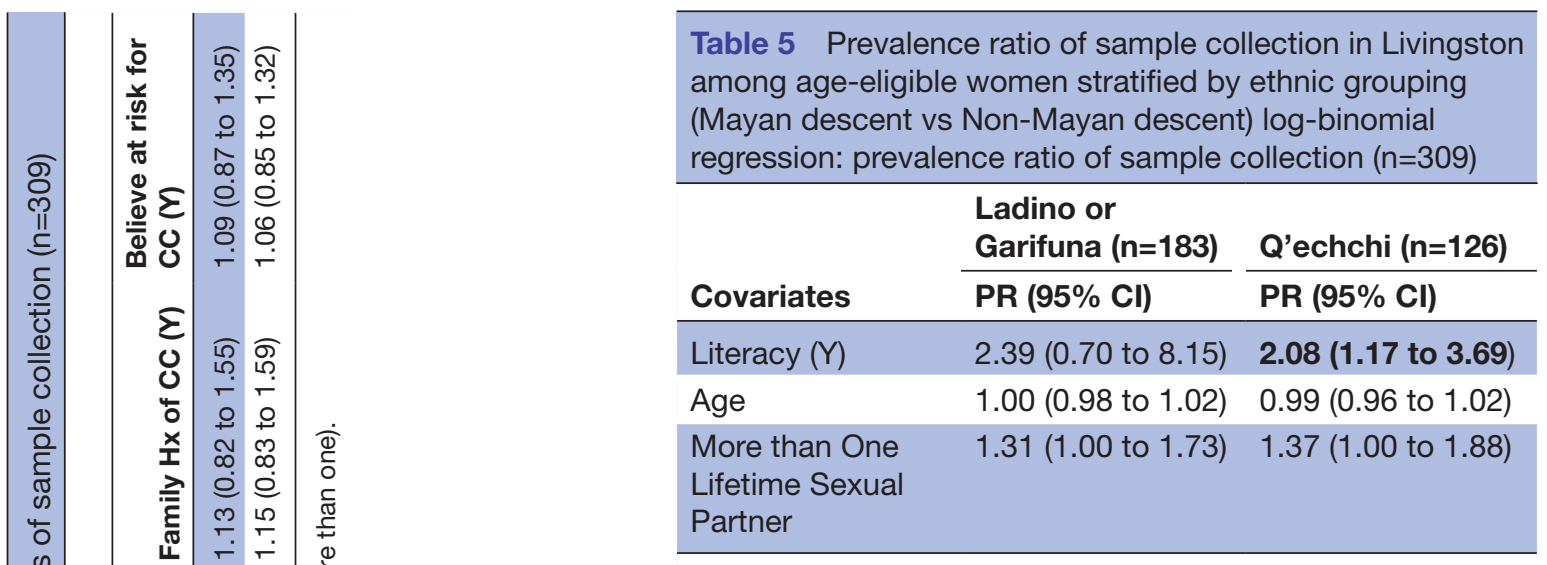

Final stepwise selected model presented, stratified by ethnic group.

Although based on a different HPV test than in our pilot study (Hybribio HR13 vs Anyplex 28), a similar prevalence of high-risk HPV was found in Santiago between 2015 and $2016(17.4 \%$ vs $118.7 \%) .{ }^{32}$ Of note, there were no significant differences in high-risk HPV prevalence between ethnic groups in Livingston, and there was not a statistically significant difference between Santiago Atitlán and Livingston with regard to prevalence.

Our study provided not only a larger sample size compared with previous studies but was also conducted in two differing communities. This is a strength because Guatemala is an extremely diverse country with over 23 languages, distinct ethnicities and a history of large economic and social inequalities. Thus, generalising the evaluations of a health programme's acceptability and feasibility to the whole country is generally difficult. However, because we evaluated two very different rural multiethnic communities, our results may reflect some of the future obstacles and considerations necessary in implementing self-swab HPV testing in such a diverse country as Guatemala than was previously available. In fact, our results also complement the findings of the ongoing careHPV Scale-Up implementation, which is assessing the performance of HPV testing, including self-collection testing, within urban settings in Guatemala. ${ }^{40-42}$

There are several limitations to our study. Due to both the sensitive nature of the questions related to sexual history, it may be possible that a social desirability bias may have resulted in over-reporting of perceived 'good behaviours', such as screening or use of protection, in addition to under-reporting of perceived 'bad behaviours', such as number of lifetime sexual partners and other sexual behaviour measures. We tried to minimise the possibility of this bias by maintaining confidentiality with participants. Also, women may report their history of screening or utilisation of healthcare resources incorrectly if they had limited information or understanding of these services. This may be exaggerated in women with low literacy and thus explain potential over-reporting of prior cervical cancer screening in Santiago Atitlán. Additionally, because sampling methods differed between 
the two communities due to the lack of reliable census counts in Livingston, there may be differences between the communities in potential selection bias into the study and more limited comparability of the results. However, our sample in Livingston is reflective of the overall population structure of the community in terms of ethnic, age and other metrics, suggesting that influential selection bias into the study might be limited. ${ }^{43}$

Screening programme implementation is a major challenge in LMIC settings, HPV self-swab testing may serve as a helpful tool in concentrating less accessible and more expensive and invasive screening modalities only towards those that are at high risk (i.e., positive for HPV). However, as the results in Livingston showed, there are many complex features related to implementing HPV screening that need to be considered before programme adoption . Due to the longitudinal component of our study, future research with our study participants will hopefully help elucidate how HPV self-collection testing may affect women's decisions to pursue further cervical cancer screening and follow-up care in their local communities after HPV testing and receiving their results. Additionally, these data may reveal other downstream facilitators or barriers to screening that might influence the overall success of HPV self-swab testing implementation in these communities.

\section{CONCLUSION}

The results of our study add to the literature on the potential of HPV self-collection testing in LMICs, demonstrating its acceptability in two very different communities in rural Guatemala. The high rates of acceptability and willingness to retake among women who self-collected in both communities suggest that once experienced, self-collection is a valid, and even a preferred, alternative to other screening modalities from the women's perspective. However, the difference in willingness to try self-collection between these communities suggests that relative literacy levels and the availability and quality of existing healthcare programme may affect attitudes towards new screening modalities. Future research should focus on increasing the generalisability of these findings by evaluating additional communities within Guatemala for differences in willingness to try self-collection sampling and further elucidate the potential barriers to accessing and utilising cervical cancer modalities, including HPV self-collection sampling.

\section{Author affiliations}

${ }^{1}$ Epidemiology, University of Michigan School of Public Health, Ann Arbor, Michigan, USA

${ }^{2}$ Center for the Prevention of Chronic Diseases, Instituto de Nutricion de

Centroamerica y Panama, Guatemala, Guatemala

${ }^{3}$ Functional Nutrition, 0xidation, and Cardiovascular Diseases Group (NFOC-Salut), Universitat Rovira i Virgili, Hospital Universitari Sant Joan, Reus, Spain

${ }^{4}$ Clinical Prevention Services, BC Centre for Disease Control, Vancouver, British Columbia, Canada

${ }^{5}$ Department of Otolaryngology-Head and Neck Surgery and Department of Pharmacology, University of Michigan, Ann Arbor, Michigan, USA
${ }^{6}$ Department of Otolaryngology Head and Neck Surgery, University of Michigan, Ann Arbor, Michigan, USA

${ }^{7}$ Laboratory of Translational Genomics, Division of Cancer, Epidemiology and Genetics, National Cancer Institute, Gaithersburg, Maryland, USA

${ }^{8}$ Cancer Epidemiology and Prevention Program, University of Michigan Rogel Cancer Center, Ann Arbor, United States

Acknowledgements We would like to thank our participants and the community health workers from Santiago Atitlán and Livingston who performed recruitment and interviews. We like to acknowledge the contribution to our study from Regina García and Amanda Agustín from the Molecular Biology laboratory at Institute of Nutrition of Central America and Panama (INCAP). Finally, we acknowledge the help and support from Dr Jaime Chumil, Leticia Toj Umul and Karen Dubois Recinos and from the NGOs Rxiin Tnamet (Santiago Atitlán) and Fundaeco (Livingston).

Contributors ARM: Planning and key intellectual contribution, data collection, sample processing, laboratory management, and test results, statistical analysis, writing and editing. AG: Planning and key intellectual contribution, data collection, sample processing, laboratory management, and test results, writing and editing. KB: Planning and key intellectual contribution, data collection, sample processing, laboratory management, and test results, editing. AP: Planning and key intellectual contribution, data collection, sample processing, laboratory management, and test results, editing. BASR: Planning and key intellectual contribution, data collection, sample processing, laboratory management, and test results, editing. CSA: Planning and key intellectual contribution, editing. GSO: Planning and key intellectual contribution, editing. TEC: Planning and key intellectual contribution, sample processing, laboratory management, and test results, editing. MP: Planning and key intellectual contribution, sample processing, laboratory management, and test results, editing. MD: Planning and key intellectual contribution, sample processing, laboratory management, and test results, editing. CM: Planning and key intellectual contribution, sample processing, laboratory management, and test results, editing. AR-A: Planning and key intellectual contribution, data collection, sample processing, laboratory management and test results, and editing. RM: planning and key intellectual contribution, data collection, sample processing, laboratory management, and test results, statistical analysis, writing and editing.

Funding This work was supported by the University of Michigan M-Cubed Program, the University of Michigan's School of Public Health Office of Global Public Health and Department of Epidemiology, The Center for the Education of Women, The Tinker Grant through the department of Latin American and Caribbean Studies, and The University of British Columbia's School of Population and Public Health and Faculty of Medicine (CIHR Foundation Scheme: Integrated Global Control and Prevention of HPV Related Diseases and Cancer grant), with in-kind support from INCAP and in part by the Intramural Research Program of the National Institutes of Health, National Cancer Institute. Dr. Meza acknowledges support from the National Cancer Institutes of Health under Award Number P30CA046592 (Rogel Cancer Center). The content is solely the responsibility of the authors and does not necessarily represent the official views of the National Institutes of Health.

Competing interests None declared.

Patient consent for publication Not required.

Ethics approval The University of Michigan Institutional Review Board (HUM00096559) and the Institute of Nutrition of Central America and Panama Institutional Review Board (MI-CIE-16-009) approved study protocols. All participants gave oral and written informed consent prior to participation in the study. The consent was documented by a signature or fingerprint of the participant, the surveyors, and a witness.

Provenance and peer review Not commissioned; externally peer reviewed.

Data availability statement Data are available on reasonable request.

Open access This is an open access article distributed in accordance with the Creative Commons Attribution Non Commercial (CC BY-NC 4.0) license, which permits others to distribute, remix, adapt, build upon this work non-commercially, and license their derivative works on different terms, provided the original work is properly cited, appropriate credit is given, any changes made indicated, and the use is non-commercial. See: http://creativecommons.org/licenses/by-nc/4.0/.

\section{REFERENCES}

1 Cuzick J, Arbyn M, Sankaranarayanan R, et al. Overview of human papillomavirus-based and other novel options for cervical 
cancer screening in developed and developing countries. Vaccine 2008;26(Suppl 10):K29-41.

2 Ginsburg O, Badwe R, Boyle P, et al. Changing global policy to deliver safe, equitable, and affordable care for women's cancers. Lancet 2017;389:871-80.

3 Petrocy A, Katz ML. Cervical cancer and HPV: knowledge, attitudes, beliefs, and behaviors among women living in Guatemala. J Health Care Poor Underserved 2014;25:624-36.

4 World Health Organization. Global cancer observatory, 2012. Available: http://globocan.iarc.fr/Default.aspx [Accessed 5 Oct 2017]

5 Bray F, Loos AH, McCarron P, et al. Trends in cervical squamous cell carcinoma incidence in 13 European countries: changing risk and the effects of screening. Cancer Epidemiol Biomarkers Prev 2005;14:677-86.

6 Mandigo M, Frett B, Laurent JR, et al. Pairing community health workers with HPV self-sampling for cervical cancer prevention in rural Haiti. Int J Gynaecol Obstet 2015;128:206-10.

7 Mittal S, Mandal R, Banerjee D, et al. HPV detection-based cervical cancer screening program in low-resource setting: lessons learnt from a community-based demonstration project in India. Cancer Causes Control 2016;27:351-8.

8 Mathers LJ, Wigton TR, Leonhardt JG. Screening for cervical neoplasia in an unselected rural Guatemalan population using direct visual inspection after acetic acid application: a pilot study. J Low Genit Tract Dis 2005;9:232-5.

9 Ministerio de Salud Pública y Asistencia Social (MSPAS) INdEI, ICF International. Encuesta Nacional de Salud Materno Infantil 20142015. Informe final. Guatemala: MSPAS/INE/ICF, 2017.

10 Denny L, de Sanjose S, Mutebi M, et al. Interventions to close the divide for women with breast and cervical cancer between lowincome and middle-income countries and high-income countries. Lancet 2017;389:861-70.

11 Curry SJ, Krist AH, Owens DK, et al. Screening for cervical cancer: US preventive services task force recommendation statement. JAMA 2018;320:674-86.

12 Ogilvie GS, van Niekerk D, Krajden M, et al. Effect of screening with primary cervical HPV testing vs cytology testing on highgrade cervical intraepithelial neoplasia at 48 months: the HPV focal randomized clinical trial. JAMA 2018;320:43-52.

13 Basu P, Mittal S, Banerjee D, et al. Diagnostic accuracy of via and HPV detection as primary and sequential screening tests in a cervical cancer screening demonstration project in India. Int $J$ Cancer 2015;137:859-67.

14 Holt HK, Zhang L, Zhao F-H, et al. Evaluation of multiple primary and combination screening strategies in postmenopausal women for detection of cervical cancer in China. Int J Cancer 2017;140:544-54.

15 Lazcano-Ponce E, Allen-Leigh B. Innovation in cervical cancer prevention and control in Mexico. Arch Med Res 2009;40:486-92.

16 Mandelblatt JS, Lawrence WF, Womack SM, et al. Benefits and costs of using HPV testing to screen for cervical cancer. JAMA 2002;287:2372-81.

17 Arbyn M, Verdoodt F, Snijders PJF, et al. Accuracy of human papillomavirus testing on self-collected versus clinician-collected samples: a meta-analysis. Lancet Oncol 2014;15:172-83.

18 Safaeian M, Kiddugavu M, Gravitt PE, et al. Comparability of selfcollected vaginal swabs and physician-collected cervical swabs for detection of human papillomavirus infections in Rakai, Uganda. Sex Transm Dis 2007:34:429-36.

19 Cremer M, Maza M, Alfaro K, et al. Scale-Up of an human papillomavirus testing implementation program in El Salvador. J Low Genit Tract Dis 2017:21:26-32.

20 Verdoodt F, Jentschke M, Hillemanns P, et al. Reaching women who do not participate in the regular cervical cancer screening programme by offering self-sampling kits: a systematic review and meta-analysis of randomised trials. Eur J Cancer 2015;51:2375-85.

21 Arrossi S, Paolino M, Thouyaret L, et al. Evaluation of scalingup of HPV self-collection offered by community health workers at home visits to increase screening among socially vulnerable under-screened women in Jujuy Province, Argentina. Implement Sci $2017 ; 12$.

22 Arrossi S, Ramos S, Straw C, et al. HPV testing: a mixed-method approach to understand why women prefer self-collection in a middle-income country. BMC Public Health 2016;16:832.
23 Arrossi S, Thouyaret L, Herrero R, et al. Effect of self-collection of HPV DNA offered by community health workers at home visits on uptake of screening for cervical cancer (the EMA study): a population-based cluster-randomised trial. Lancet Glob Health 2015;3:e85-94.

24 Broquet C, Triboullier D, Untiet S, et al. Acceptability of self-collected vaginal samples for HPV testing in an urban and rural population of Madagascar. Afr Health Sci 2015;15:755-61.

25 Kobetz E, Seay J, Amofah A, et al. Mailed HPV self-sampling for cervical cancer screening among underserved minority women: study protocol for a randomized controlled trial. Trials 2017;18:19.

26 Ma'som M, Bhoo-Pathy $\mathrm{N}$, Nasir $\mathrm{NH}$, et al. Attitudes and factors affecting acceptability of self-administered cervicovaginal sampling for human papillomavirus (HPV) genotyping as an alternative to Pap testing among multiethnic Malaysian women. BMJ Open 2016;6:e011022.

27 Nelson EJ, Maynard BR, Loux T, et al. The acceptability of selfsampled screening for HPV DNA: a systematic review and metaanalysis. Sex Transm Infect 2017;93:56-61.

28 Silva J, Cerqueira F, Medeiros R. Acceptability of self-sampling in Portuguese women: the good, the bad or the ugly? Sex Health 2017;14:298-300.

29 Vahabi M, Lofters A. Muslim immigrant women's views on cervical cancer screening and HPV self-sampling in Ontario, Canada. BMC Public Health 2016;16:868.

30 Williams D, Davies M, Fiander A, et al. Women's perspectives on human papillomavirus self-sampling in the context of the UK cervical screening programme. Health Expect 2017;20:1031-40.

31 Wong ELY, Cheung AWL, Huang F, et al. Can human papillomavirus DNA self-sampling be an acceptable and reliable option for cervical cancer screening in female sex workers? Cancer Nurs 2018:41:45-52.

32 Gottschlich A, Rivera-Andrade A, Grajeda E, et al. Acceptability of human papillomavirus self-sampling for cervical cancer screening in an Indigenous community in Guatemala. J Glob Oncol 2017;3:444-54.

33 Ministerio de Salud Pública y Asistencia SocialPlan nacional de prevención. Plan nacional de prevención, control Y manejo de cáncer cervicouterino 2014-2024. Guatemala: Ministerio de Salud Pública y Asistencia Social Plan nacional de prevención, 2014.

34 El-Zein M, Bouten S, Louvanto K, et al. Validation of a new HPV selfsampling device for cervical cancer screening: the cervical and selfsample in screening (CASSIS) study. Gynecol Oncol 2018;149:491-7.

35 Eve Medical. Improving sexual health screening: HerSwab, 2018. Available: http://www.eve-medical.com/.

36 Lou H, Gharzouzi E, Guerra SP, et al. Low-Cost HPV testing and the prevalence of cervical infection in asymptomatic populations in Guatemala. BMC Cancer 2018;18:562.

37 Castle PE, Sadorra M, Garcia FAR, et al. Mouthwash as a low-cost and safe specimen transport medium for human papillomavirus DNA testing of cervicovaginal specimens. Cancer Epidemiol Biomarkers Prev 2007;16:840-3

38 Fokom Domgue J, Schiffman M, Wentzensen NH, et al. Assessment of a new lower-cost real-time PCR assay for detection of high-risk human papillomavirus: useful for cervical screening in limitedresource settings? J Clin Microbiol 2017;55:2348-55.

39 Hybridbio. 13 high-risk HPV real-time PCR kit, 2017. Available: http:// hybribio.com/content/portfolio-view/13-high-risk-hpv-real-time-pcrkit/ [Accessed 4 Apr 2017].

40 Holme F, Kapambwe S, Nessa A, et al. Scaling up proven innovative cervical cancer screening strategies: challenges and opportunities in implementation at the population level in low- and lower-middleincome countries. Int J Gynecol Obstet 2017;138:63-8.

41 Jeronimo J, Bansil P, Lim J, et al. A multicountry evaluation of careHPV testing, visual inspection with acetic acid, and Papanicolaou testing for the detection of cervical cancer. Int J Gynecol Cancer 2014;24:576-85

42 Jeronimo J, Holme F, Slavkovsky R, et al. Implementation of HPV testing in Latin America. J Clin Virol 2016;76(Suppl 1):S69-73.

43 Proyección de Poblaciones por Área 2016. Guatemala Ministerio de Salud Pública y Asistencia Social, Departamento de Epidemiología; 2016. http://epidemiologia.mspas.gob.gt/informacion/estadisticasvitales/poblacion-y-proyeccion 